\title{
Alteraciones tiroideas en la infancia y en la adolescencia. Parte 1: hipertiroidismo
}

Thyroid disorders in childhood and adolescence. Part 1: Hyperthyroidism

\author{
Comité Nacional de Endocrinología de la Sociedad Argentina de Pediatría
}

\section{RESUMEN}

Elhipertiroidismo es el cuadro clínico resultante del exceso de hormonas tiroideas debido a hiperfunción glandular. Es una enfermedad rara en niños y adolescentes, pero con una alta morbilidad. La causa más frecuente es la enfermedad de Graves. El objetivo de esta publicación es realizar una revisión y actualización del hipertiroidismo infantojuvenil para guiar su detección y derivación temprana al endocrinólogo pediatra. Debe ser considerado cuando el niño o adolescente presente síntomas asociados a esta patología y bocio de grado variable. Se confirma con el perfil bioquímico característico.

El tratamiento consiste, inicialmente, en bloquear los efectos del exceso de hormonas tiroideas con betabloqueantes y, además, disminuir su producción con drogas antitiroideas como primera elección. Ante efectos secundarios a su administración, recidivas o ausencia de remisión de la enfermedad, se optará por el tratamiento definitivo: yodo radioactivo o cirugía con el objetivo de lograr el hipotiroidismo o eutiroidismo.

Palabras clave: hipertiroidismo, enfermedad de Graves, antitiroideos, niño, adolescente.

\begin{abstract}
Hyperthyroidism is a serious and rare disorder in childhood characterized by the overproduction of thyroid hormones by the thyroid gland. Graves disease is the most common cause. The objective of this paper is to review and update hyperthyroidism in children and adolescents aiming to guide its early detection and referral to the pediatric endocrinologist. The disease should be suspected if typical symptoms and goiter are present and has to be confirmed with the characteristic biochemical profile. Initially, treatment to block the effect of the thyroid excess is needed. Antithyroid drugs are the recommended first-line treatment to diminish hormone production. Alternative treatments, such us radioactive iodine or thyroidectomy, are
\end{abstract}

Dra. María S. Rodríguez Azrak:

solazrak@gmail.com

\section{Financiamiento:}

Ninguno.

Conflicto de intereses:

Ninguno que declarar.

Recibido: 23-12-2019

Aceptado: $12-8-2020$ considered in cases of adverse effects to drugs, relapse or non-remission of the disease, in order to achieve hypothyroidism or euthyroidism. Key words: hyperthyroidism, Graves disease, antithyroid agents, child, adolescent.

http:/ / dx.doi.org/10.5546/ aap.2021.S1

Cómo citar: Comité Nacional de Endocrinología. Alteraciones tiroideas en la infancia y en la adolescencia. Parte 1: hipertiroidismo. Arch Argent Pediatr 2021;119(1):S1-S7.

\section{GENERALIDADES}

Durante la infancia, las hormonas tiroideas juegan un rol crítico en el crecimiento, desarrollo y metabolismo, pues modulan la función de la mayoría de los sistemas del organismo. Las consecuencias de sus alteraciones incluyen efectos a nivel cardiovascular, neurológico, cognitivo, gastrointestinal y metabólico.

La disfunción tiroidea en el niño y en el adolescente puede tener múltiples causas, de las cuales la más frecuente es la enfermedad autoinmune. Esta engloba un amplio espectro de disfunciones, que comprenden desde el hipotiroidismo, frecuentemente asociado a la tiroiditis linfocitaria crónica (TLC) hasta el hipertiroidismo de la enfermedad de Graves (EG), y es mucho más frecuente la TLC (relación $7: 3){ }^{1}$
Primera autora: Dra. Sonia V. Bengolea

Dr. Guillermo Alonso, Dra. Andrea Arcari, Dra. Elisabeth Boulgourdjian,

Dra. Mariana Costanzo, Dra. Silvia D’Amato, Dra. Ana Keselman, Dra. Silvia Martin, Dra. Viviana R. Pipman, Dra. María S. Rodríguez Azrak, Dra. Clara Valeri. 
El objetivo de esta publicación es realizar una revisión y actualización de la disfunción tiroidea en la población infantil para su detección y derivación temprana al endocrinólogo. En esta primera parte, se actualizará sobre el hipertiroidismo en la infancia y en la adolescencia.

\section{INTRODUCCIÓN}

El hipertiroidismo es el cuadro clínico resultante del exceso de hormonas tiroideas debido a hiperfunción glandular, mientras que el término tirotoxicosis se refiere a las manifestaciones clínicas y bioquímicas del exceso de hormonas tiroideas. ${ }^{1}$ La mayoría de los casos pediátricos son por hipertiroidismo y es rara la tirotoxicosis secundaria a una tiroiditis destructiva o a causas extratiroideas ${ }^{2}$ (Tabla 1).

\section{Epidemiología y etiología}

El hipertiroidismo en la infancia es una enfermedad rara; corresponde al $15 \%$ de las alteraciones tiroideas pediátricas ${ }^{3}$ y es menos frecuente que en los adultos. Se presenta con una incidencia anual de 8/1000 000 en los menores de 15 años y de 1/1000 000 en los menores de 4 años. ${ }^{4}$ Las causas pediátricas de hipertiroidismo se enumeran en la Tabla 1; la más frecuente es la enfermedad de Graves (EG), que corresponde al $84 \%$ de los casos. ${ }^{5,6}$

\section{Enfermedad de Graves}

La EG es una patología autoinmune caracterizada por la estimulación del receptor de tirotrofina (thyroid stimulating hormone; TSH, por sus siglas en inglés) por autoanticuerpos estimulantes (thyrotropin-receptor antibody; TRAb, por sus siglas en inglés). Si bien puede presentarse a partir de los 3 años, la mayor incidencia es en la etapa peripuberal, entre los 9 y los 13 años, con una mayor prevalencia a esta edad en el sexo femenino (relación F/M: 5,8/1), mientras que, en los niños más pequeños, la relación es $1: 1 .^{1,2}$

Los niños con EG pueden presentar antecedentes familiares de patología tiroidea autoinmune $\mathrm{e}^{7} \mathrm{y}$ asociarse a otras enfermedades autoinmunes, como artritis reumatoidea juvenil, anemia perniciosa, lupus eritematoso sistémico, enfermedad de Addison, enfermedad celíaca y vitiligo. ${ }^{8}$ También es más frecuente en los niños con síndrome de Down, Turner y Di George. ${ }^{9,10}$

\section{Etiopatogenia}

La patogenia es poco clara; resulta de una compleja interacción entre factores genéticos (HLA DR3, B8; CTLA-4; PTPN22), ambientales (estrés, infecciones, fármacos, tabaquismo, etc.) y el sistema inmune. ${ }^{4}$ También existen condiciones emocionales desencadenantes (mudanzas, muertes cercanas, etc.). La infiltración linfocitaria, con aumento de linfocitos T CD4+ helpers y disminución de la actividad de linfocitos supresores, con secreción de citoquinas y la activación inflamatoria local, lleva a una desregulación de la actividad de los linfocitos B y a un aumento en la producción de autoanticuerpos ${ }^{6}$ (Figura 1).

TABLA 1. Causas de hipertiroidismo en la infancia y en la adolescencia ${ }^{5}$

- Enfermedad de Graves.

- Hipertiroidismo neonatal (por pasaje transplacentario de anticuerpos maternos estimulantes de la tiroides: TRAb).

- Tiroiditis.

* Tiroiditis aguda destructiva -tirotoxicosis .

* Tiroiditis subaguda.

* Tiroiditis linfocitaria crónica o enfermedad de Hashimoto (fase hipertiroidea).

- Nódulos autónomos funcionantes.

* Mutación somática activante de Gs $\alpha$ : síndrome de McCune-Albright.

* Mutación somática activante del gen del receptor de TSH.

* Adenoma tóxico.

* Carcinoma papilar o folicular hiperfuncionante.

- Mutación congénita activante del gen del receptor de TSH (hereditaria o de novo): hipertiroidismo congénito.

- Resistencia a las hormonas tiroideas.

- Tumor pituitario secretor de TSH.

- Causas exógenas.

* Ingesta de hormonas tiroideas (aguda o crónica): tirotoxicosis .

* Hipertiroidismo inducido por yodo (yodo, agentes de contraste, amiodarona). 


\section{Manifestaciones clínicas}

Los signos y síntomas de la EG en los niños y en los adolescentes son similares a los de los adultos, con efectos únicos sobre el crecimiento y el desarrollo puberal; ${ }^{1}$ son excepcionales la oftalmopatía maligna y el edema pretibial. ${ }^{1} \mathrm{La}$ enfermedad comienza en forma insidiosa, con la instalación del cuadro florido a lo largo de varios meses. ${ }^{11}$

Los pacientes pueden presentar lo siguiente:

- Cambios de conducta, hiperquinesia, nerviosismo, irritabilidad, fatiga, labilidad emocional, dificultad en la concentración, alteraciones del rendimiento escolar.

- Palpitaciones.

- Temblor de los miembros y de la lengua (fasciculaciones).

- Hiperfagia acompañada de pérdida de peso, diarreas, crecimiento acelerado. ${ }^{11}$

- Cansancio muscular proximal.

- Alteración del ciclo menstrual.

En la exploración física, se destacan bocio (en el $98 \%$ de los casos), en general, de tamaño moderado, difuso, sin nodularidad, simétrico, firme e indoloro, ${ }^{11,12}$ y compromiso sistémico: piel suave, fina, húmeda, caliente y sudorosa, temblor fino distal, taquicardia sinusal de grado variable, hipertensión arterial con mayor amplitud de la tensión arterial; ${ }^{12}$ descenso de peso y aceleración del crecimiento prepuberal.

A diferencia de los adultos, solo $1 / 3$ de los pacientes pediátricos presenta oftalmopatía, que es típicamente moderada, sin riesgo de pérdida de la visión. El compromiso ocular, presente, sobre todo, al inicio del cuadro, es, por lo general, secundario al aumento del tono adrenérgico y mejora con la remisión de la enfermedad., ${ }^{4,713}$

El diagnóstico de la EG, a veces, se retrasa, debido a que no se considera el hipertiroidismo como causa de los síntomas del paciente, que son atribuidos a otras patologías. Es por ello por lo que es importante considerar el hipertiroidismo como diagnóstico diferencial en un paciente con alteraciones de la conducta, ansiedad o déficit de atención e hiperactividad, asma inducida por el ejercicio o una arritmia cardíaca primaria. ${ }^{10}$

Un breve comentario merece la tormenta tiroidea o crisis tirotóxica, que es un evento agudo y grave, potencialmente fatal, debido a la exacerbación del hipertiroidismo. Ocurre siempre en el contexto de un hipertiroidismo no controlado. Algunos desencadenantes de las crisis son cirugía, infecciones, trauma, cetoacidosis diabética o aporte excesivo de yodo. Es considerada una emergencia endocrinológica; requiere internación y tratamiento agresivo.

\section{Diagnóstico}

Tras la presunción clínica, el diagnóstico bioquímico confirmará el hipertiroidismo con niveles suprimidos de TSH y, generalmente, niveles elevados de hormonas tiroideas: tiroxina (T4), tiroxina libre (T4L) y triyodotironina (T3). En la mayoría de los pacientes con EG, se detectan anticuerpos antitiroglobulina y antiperoxidasa positivos. El anticuerpo más importante para el diagnóstico es el que ocupa el receptor de TSH y lo estimula: $T R A b b^{1,11}$

La ecografía tiroidea puede ser de utilidad para el diagnóstico etiológico. Descartará la

FIGURA 1. Fisiopatología del hipertiroidismo autoinmune

Fisiopatología multifactorial

Factores inmunológicos

Linfocitos $\mathrm{T}$ anormales

Citoquinas

$$
\downarrow
$$

- Inflamación local

- Remodelación tisular

- Aumento de producción de

autoanticuerpos receptor TSH (TRAB)
Factores genéticos

Poligénico

- Antígenos HLA (cromosoma 6)

- Linfocitos T citotóxicos (CTLA-4, cromosoma 2q33)

- Gen de la fosfatasa tirosina linfoide (PTPN22, cromosoma 1p13).
Factores ambientales

Medicamentos

Tabaco

Infecciones

Estrés

Situaciones dolorosas afectivas:

- Muerte.

- Mudanzas.

- Accidentes 
presencia de nódulos y mostrará los hallazgos ecográficos de EG, como aumento del volumen tiroideo, ecoestructura heterogénea e hipervascularización (en ecografía con Doppler). ${ }^{10}$ Por su parte, el centellograma tiroideo estará indicado solo si se presenta un nódulo palpable o visible en la ecografía. ${ }^{4}$

\section{Diagnósticos diferenciales}

El diagnóstico diferencial de EG debe realizarse con otras causas clínicas de la sintomatología del paciente (cardíacas, infecciosas y emocionales) jerarquizando, sobre todo, en favor de EG la presencia de bocio. Este está también presente en algunas otras causas menos comunes de hipertiroidismo, como la fase hipertiroidea de la tiroiditis linfocitaria crónica, la tiroiditis subaguda (excepcional en la infancia) y otras formas de hipertiroidismo no autoinmune.

El bocio uni- o multinodular hipertiroideo tóxico es muy raro en pediatría. Más allá de la evaluación de la función tiroidea, en los pacientes con estos hallazgos, se deben realizar ecografía y centellograma tiroideo. ${ }^{2}$

El síndrome de McCune-Albright (displasia fibrosa poliostótica) puede incluir entre sus manifestaciones endócrinas la autonomía tiroidea con hipertiroidismo. La resistencia a las hormonas tiroideas, generalmente, ocurre en $1: 50000$ niños y los afecta con un cuadro de manifestaciones específicas del órgano de hiper- e hipotiroidismo con un perfil bioquímico de hormonas tiroideas altas con TSH normal o ligeramente elevada. Su sospecha requiere la derivación al especialista. En la Tabla 2, se describen las características principales de los diagnósticos diferenciales de hipertiroidismo y su orientación terapéutica.

\section{TRATAMIENTO}

El abordaje del niño y del adolescente hipertiroideo requiere del tratamiento inicial para atenuar los síntomas del exceso de hormonas tiroideas y del tratamiento dirigido a disminuir la producción de estas por la glándula. Una vez confirmado el diagnóstico, la evaluación cardiológica es de rigor para definir la necesidad del betabloqueo y excluir trastornos previos de la conducción cardíaca que pudieran contraindicarlo. Este será necesario cuando exista taquicardia sinusal franca, y el aleteo o fibrilación es el criterio de internación.

El propranolol será el fármaco de elección (1-2 mg/ kg/día) para mejorar los síntomas adrenérgicos. ${ }^{1}$ En los niños con antecedentes de broncoespasmo o asma, se utilizan fármacos más cardioselectivos, como el atenolol (25-50 mg/ día). Se contraindicará la actividad física hasta lograr el eutiroidismo.

TABla 2. Diagnósticos diferenciales, prevalencia y tratamiento

\begin{tabular}{|c|c|c|c|c|c|c|}
\hline Patología & TSH & T4 libre & $T R A b$ & АТРО & Prevalencia $(\%)$ & Terapia \\
\hline Enfermedad de Graves & $\downarrow$ & $\uparrow$ & + & + & 84 & $\begin{array}{c}\text { Drogas antitiroideas } \\
\text { Radioyodo } \\
\text { Tiroidectomía }\end{array}$ \\
\hline $\begin{array}{l}\text { Fase hipertiroidea } \\
\text { de tiroiditis autoinmune } \\
\text { (Hashitoxicosis) }\end{array}$ & $\downarrow$ & $\uparrow$ & - & + & 12 & Drogas antitiroideas \\
\hline $\begin{array}{l}\text { Nódulos } \\
\text { tiroideos hiperfuncionantes }\end{array}$ & $\downarrow$ & $\mathrm{No} \uparrow$ & - & & $<3$ & Tiroidectomía \\
\hline $\begin{array}{l}\text { Adenoma hipofisario } \\
\text { productor de TSH }\end{array}$ & $\uparrow$ & $\mathrm{No} \uparrow$ & - & & $\approx 1$ & Cirugía \\
\hline $\begin{array}{l}\text { Mutaciones activantes } \\
\text { del receptor de TSH }\end{array}$ & $\downarrow$ & $\uparrow$ & - & & $\approx 1$ & Tiroidectomía \\
\hline $\begin{array}{l}\text { Síndrome de } \\
\text { McCune-Albright }\end{array}$ & $\downarrow$ & No $\uparrow$ & - & & & $\begin{array}{c}\text { Drogas antitiroideas } \\
\text { o tiroidectomía }\end{array}$ \\
\hline $\begin{array}{l}\text { Resistencia a las } \\
\text { hormonas tiroideas }\end{array}$ & $\uparrow$ o N & $\uparrow$ & - & & & $\begin{array}{c}\beta \text {-bloqueantes } \\
\text { Ácido 3,5,3'-triyodo- } \\
\text { tiroacético (TRIAC) }\end{array}$ \\
\hline
\end{tabular}

TSH: hormona estimulante de la tiroides; TRAb: anticuerpos contra el receptor de tirotrofina; ATPO: anticuerpos antiperoxidasa tiroidea; N: normal. 
De allí en más, el tratamiento debe ser realizado o guiado por un endocrinólogo pediatra, que utilizará como opción terapéutica los fármacos antitiroideos o se decidirá por tratamientos definitivos, como radioyodo o cirugía. Ninguno de ellos cumple con criterios absolutos de seguridad y efectividad, y cada uno tiene sus ventajas y desventajas. La elección se basará, fundamentalmente, en la situación del paciente y en la experiencia del médico tratante. ${ }^{14,15}$ En la Tabla 3, se describen las opciones terapéuticas, sus dosis y eventos adversos.

\section{Drogas antitiroideas}

Dentro de los fármacos antitiroideos, el metimazol o metilmercaptoimidazol es el tratamiento de primera línea. Es el único disponible en nuestro país y también el único recomendado en la edad pediátrica. El propiltiouracilo, en la actualidad, está contraindicado por el riesgo de hepatotoxicidad en los niños, dado que hay reportes de necrosis y falla hepática fulminante. ${ }^{9,12}$ Su utilización queda reservada solo para el embarazo, debido a los efectos teratogénicos del metilmercaptoimidazol.

Las drogas antitiroideas inhiben la síntesis de hormonas tiroideas e interfieren en la oxidación y organificación del yodo. No actúan sobre la liberación de las hormonas tiroideas ya formadas, motivo por el cual la función tiroidea se normaliza solo después de varias semanas de tratamiento (68 semanas), en las cuales el tratamiento de sostén deberá ser mantenido.

La dosis media inicial de metilmercaptoimidazol recomendada es de $0,5 \mathrm{mg} / \mathrm{kg} /$ día dependiendo de la gravedad del hipertiroidismo ${ }^{10}$ (e intentando no superar los $30 \mathrm{mg} /$ día). Antes de comenzar el tratamiento, se realizará un hepatograma y hemograma para documentar el recuento de glóbulos blancos y el valor de transaminasas iniciales, en vista de los posibles efectos adversos del metilmercaptoimidazol.

Las familias y los pacientes deben recibir información sobre los probables efectos adversos. En caso de presentarse, suelen aparecer, durante los primeros 6 meses de tratamiento, urticaria, rash, dolor articular y fatiga, que, en general, son bien tolerados, transitorios y no es necesario suspender el tratamiento. ${ }^{10}$ Los efectos adversos graves son poco frecuentes: neutropenia, artritis, hepatitis, que obligan a interrumpir el tratamiento. ${ }^{6}$ Si presentaran ictericia, fiebre o faringitis, se debe realizar un hemograma (evaluación de glóbulos blancos) y una evaluación hepática para constatar la adecuada respuesta medular y descartar el compromiso hepático farmacodependiente.

El control del paciente hipertiroideo será frecuente durante el primer año de tratamiento.

TABLA 3. Opciones terapéuticas, dosis y eventos adversos

\begin{tabular}{|c|c|c|}
\hline Tratamiento & Dosis & Eventos adversos \\
\hline \multicolumn{3}{|c|}{ Médico: } \\
\hline \multicolumn{3}{|c|}{ Drogas antitiroideas: } \\
\hline Metimazol & 0,2-1 mg/kg/día. & $\begin{array}{l}\text { Leves: urticaria, rash, dolor articular y fatiga. } \\
\text { Graves: neutropenia, artritis y hepatitis. }\end{array}$ \\
\hline Propiltiouracilo & Contraindicado. & Insuficiencia hepática. \\
\hline \multicolumn{3}{|l|}{ Betabloqueantes: } \\
\hline Propanolol & 1-2 mg/kg/día. & Broncoespasmo, arritmias. \\
\hline Atenolol & 25-50 mg/ día. & Arritmias. \\
\hline Radioyodo & $\begin{array}{l}\text { Calculada según el tamaño } \\
\text { de la glándula } \\
\text { y el valor de la captación a las } 24 \text { h } \\
\text { en la cara anterior del cuello. }\end{array}$ & $\begin{array}{l}\text { Leves: náuseas y dolor local } \\
\text { Crisis tirotóxica. } \\
\text { Contraindicado en las embarazadas } \\
\text { (riesgo de teratogenicidad) y en los niños } \\
\text { menores de } 5 \text { años; preferentemente, } \\
\text { en los mayores de } 10 \text { años. }\end{array}$ \\
\hline Tiroidectomía & Total. En estado de eutiroidismo. & $\begin{array}{c}\text { Crisis tirotóxica. } \\
\text { Hipocalcemia, hematomas, } \\
\text { hipoparatiroidismo permanente y } \\
\text { lesiones del nervio laríngeo recurrente. }\end{array}$ \\
\hline
\end{tabular}


El especialista, por lo general, titula la dosis del antitiroideo hasta lograr el eutiroidismo bajo la mínima dosis posible de medicación. Dado que la enfermedad pediátrica tiene un curso largo con una remisión del $25 \%$ cada 2 años, se realiza un primer tratamiento de 2-3 años de duración con controles clínico-bioquímicos y, luego, de acuerdo con la persistencia o no de marcadores de enfermedad, se suspende el tratamiento o se continúa por $2 / 3$ años más.

Se considera remisión al logro del eu- o hipotiroidismo de, por lo menos, 1 año desde la suspensión de la terapia. La tasa de remisión de la población pediátrica a 10 años es de, aproximadamente, el $70 \%$. Algunos factores relacionados con peores tasas de remisión son sexo masculino, menor edad al momento del diagnóstico, bocio grande, pacientes prepúberes, menor índice de masa corporal, oftalmopatía, recidivas previas y niveles muy elevados iniciales de hormonas tiroideas o de TRAb. ${ }^{16}$

La adherencia al tratamiento es muy importante en el manejo de estos niños y debería ser mejorada por estrategias educacionales. En este sentido, toma relevancia el papel del pediatra, que atiende otros aspectos de su salud. La visita pediátrica en los pacientes con esta enfermedad debería incluir un interrogatorio sobre la adherencia a la indicación y un refuerzo de esta conducta. La comunicación fluida con el especialista facilitará el manejo.

\section{TRATAMIENTO DEFINITIVO}

Son indicados, como se mencionó anteriormente, ante la imposibilidad de continuar con las drogas antitiroideas.

\section{Yodo radioactivo}

Como tratamiento de segunda línea y de acuerdo con su disponibilidad, es el más frecuentemente elegido. La tasa de remisión con este tratamiento es elevada (del 70-95\%). Por lo general, se alcanza con una sola toma oral y puede repetirse a los seis meses de ser necesario. El objetivo del tratamiento es inducir el hipotiroidismo.

Su administración no está recomendada en los niños menores de 5 años y, sobre todo, se indica en los mayores de 10 años. Está totalmente contraindicado en las mujeres embarazadas y durante la lactancia. También suele contraindicarse en los pacientes con oftalmopatía grave (infrecuente en pediatría). Los pacientes que reciben I-131 deben guardar precauciones de aislamiento y evitar el contacto con otros niños y embarazadas de acuerdo con lo indicado por el especialista.

\section{Cirugía}

Es el tratamiento de segunda línea recomendado en bocios grandes, nodulares, en niños pequeños o con grave oftalmopatía. ${ }^{14}$ La tiroidectomía total es preferida a la cirugía subtotal para disminuir el riesgo de recurrencia del hipertiroidismo. ${ }^{10}$ Debe realizarse por cirujanos expertos en patología tiroidea (más de 30 tiroidectomías/año) para reducir el riesgo de complicaciones. ${ }^{14}$

Antes de la cirugía, el paciente debe encontrarse eutiroideo con el objeto de prevenir una posible crisis tiroidea. En algunas oportunidades y con los debidos recaudos, puede requerirse el tratamiento previo con gotas de Lugol, que, además, disminuye la vascularización de la glándula. ${ }^{14}$ El tratamiento hormonal sustitutivo con levotiroxina si el bocio no es nodular debe comenzar inmediatamente después de la cirugía. Las posibles complicaciones de la cirugía incluyen hipocalcemia, hematomas, hipoparatiroidismo permanente y lesiones del nervio laríngeo recurrente. ${ }^{14}$

\section{REFERENCIAS}

1. Iorcansky S. Hipertiroidismo Infanto-juvenil. En: Gauna A, Novelli JL, Sanchez A (eds.). Hipertiroidismo. Rosario: Universidad Nacional de Rosario; 2008.Págs.209-16.

2. LaFranchi S. Clinical manifestations and diagnosis of Graves disease in children and adolescents. UpToDate. [Acceso: julio de 2018]. Disponible en: https://www. uptodate.com / contents / clinical-manifestations-anddiagnosis-of-graves-disease-in-children-and adolescents.

3. Busiah K, Giabicani E. Hipotiroidismo e Hipertiroidismo infantil. EMC-Tratado de Medicina. 2017; 21(4):1-9.

4. Zirilli G, Velletri MR, Porcaro F, Di Giovine G, et al. Hyperthyroidsm in childhood: peculiarities of the different clinical pictures. Acta Bio Med. 2015; 86(3):220-5.

5. De Lucca F, Salzano G, Zirilli G, Calafiore M, et al. Management of Hyperthiroidism in Children. Expert Rev Endocrinol Metab. 2016; 11(4):301-9.

6. Leger J, Carel JC. Hyperthyroidism in childhood: causes, when and how to treat. J Clin Res Pediatr Endocrinol. 2013; 5(Suppl 1):50-6.

7. Cappa M, Bizzarri C, Crea F. Autoinmune thyroid diseases in children. J Thyroid Res. 2011; 2011:675703.

8. Boelaert K, Newby PR, Simmonds MJ, Holder RL, et al. Prevalence and relative risk of other autoimmune diseases in subjects with autoimmune thyroid disease. Am J Med. 2010; 123(2):183.e1-9.

9. De Luca F, Corrias A, Salerno M, Wasniewska M, et al. Peculiarities of Graves' disease in children and adolescents with Down's syndrome. Eur J Endocrinol. 2010; 162(3):591-5.

10. Léger J, Carel JC. Diagnosis and management of hyperthyroidism from prenatal life to adolescence. Best Pract Res Clin Endocrinol Metab. 2018; 32(4):373-86. 
11. RivkeesSA.Hyperthyroidism, Childhood and Adolescence. In: Huhtaniemi I (ed.). Encyclopedia of Endocrine Diseases. 2nd ed. Oxford: Academic Press; 2018.Págs.317-27.

12. SrinivasanS, Misra M.Hyperthyroidism in Children. Pediatr Rev. 2015; 36(6):239-48.

13. Goldstein SM, Katowitz WR, Moshang T, Katowitz JA. Pediatric thyroid-associated orbitopathy: the Chlidren's Hospital of Philadelphia experience and literatura review. Thyroid. 2008; 18(9):997-9.
14. Chiesa A, Gruñeiro-Papendieck L. Tratamiento y seguimiento del hipertiroidismo en la infancia. En: Gauna A, Novelli JL, Sanchez A (eds.). Hipertiroidismo. Rosario: Universidad Nacional de Rosario; 2008.Págs.217-26.

15. Gruñeiro-Papendieck L, Chiesa A, Finkielstain A, Heinrich JJ. Pediatric Graves' disease: outcome and treatment. J Pediatr Endocrinol Metabol. 2003; 16(9):1249-55.

16. Glaser NS, Styne DM. Predictors of early remission of hyperthyroidism in children. J Clin Endocrinol Metab. 1997; 82(6):1719-26. 